\title{
Caractérisation des protéines de choc thermique de faible poids moléculaire chez les bactéries lactiques
}

\author{
Michel-Philippe Jobin, Françoise Delmas, Dominique Garmyn, \\ Charles Divies, Jean Guzzo
}

Laboratoire de microbiologie, U.A.-Inra, Ensbana, esplanade Erasme, 21000 Dijon, France

\begin{abstract}
Characterization of small heat shock proteins in lactic acid bacteria. Among heat shock proteins (Hsp), low molecular weight Hsp (smHsp) are the less known. Four smHsp were recently studied in lactic acid bacteria (Streptococcus thermophilus, Lactobacillus delbrueckii, Lactobacillus helveticus et Oenococcus oeni). These smHsp are related to Eukaryotic $\alpha$-crystallins able to form high molecular weight complexes with a chaperoning activity. Induction factors of smHsp vary with the lactic acid bacterium. The expression of $L$. helveticus and $O$. oeni $\mathrm{smHsp}$ genes are governed by different promoter structures. In $L$. helveticus, the regulation system is like Lactococcus lactis stress genes already known with a vegetative promoter and a CIRCE sequence. In $O$. oeni, the regulation seems to be closely related to the class III stress genes, the less known mechanism of regulation in Bacillus subtilis. (C) Inra/Elsevier, Paris.
\end{abstract}

stress / lactic acid bacteria / smHsp / $\alpha$-crystallin

Résumé - Parmi les protéines de choc thermique (Hsp), celles de faible poids moléculaire (smHsp) sont les moins connues. Quatre smHsp ont été récemment étudiées chez des bactéries lactiques (chez Streptococcus thermophilus, Lactobacillus delbrueckii, Lactobacillus helveticus et Oenococcus oeni). Ces smHsp sont apparentées aux $\alpha$-cristallines des Eucaryotes capables de former des complexes de haut poids moléculaire possédant une activité de chaperonne. Les facteurs d'induction des smHsp varient selon les bactéries lactiques. L'expression des gènes de smHsp de $L$. helveticus et d'O. oeni est gouvernée par des structures promotrices différentes. Chez $L$. helveticus le système de régulation serait comparable aux gènes de stress connus chez Lactococcus lactis, avec un promoteur végétatif et un séquence CIRCE. Alors que chez O. oeni, la régulation serait plus proche du modèle encore méconnu des gènes de stress de classe III décrit chez Bacillus subtilis. (C) Inra/Elsevier, Paris.

stress / bactérie lactique / smHsp / $\alpha$-cristalline 


\section{INTRODUCTION}

Les bactéries lactiques sont capables de fermenter les sucres en acide lactique. Leur capacité de tolérance à l'acidité en fait un outil de technologie industrielle pour la transformation et la conservation de nombreux produits alimentaires d'origine animale ou végétale. Lors des procédés industriels, les bactéries lactiques sont soumises à des stress multiples pouvant provenir de la croissance bactérienne elle-même (par acidification du milieu et appauvrissement en nutriments) ou imposés dans l'environnement (par modification de la température ou du $\mathrm{pH}$, choc osmotique, lyophilisation, etc).

Les bactéries possèdent cependant un pouvoir d'adaptation. Un choc physicochimique sublétal entraîne la mise en place de dispositifs physiologiques temporaires assurant la survie dans le nouvel environnement. L'étude de ces mécanismes de réponse aux stress chez les bactéries lactiques devrait permettre d'améliorer la qualité des levains lactiques utilisés en industrie.

Oenococcus oeni, anciennement Leuconostoc oenos [6], présente des propriétés acidophiles et de résistance à l'éthanol lui permettant de résister aux conditions environnementales extrêmes du vin (un $\mathrm{pH}$ allant de 3 à 3,5 , des concentrations en éthanol de 10 à $13 \%$ et en sulfites de 50 à $100 \mathrm{mg} / \mathrm{L}$ ). Cette bactérie apparaît comme un bon modèle pour étudier la réponse à de multiples stress chez les bactéries lactiques.

En se développant dans le vin, $O$. oeni réalise la fermentation malolactique (FML) qui est la décarboxylation du L-malate en L-lactate. Cette désacidification qui intervient après la fermentation alcoolique, joue un rôle important sur la qualité finale de certains vins [16]. Des levains malolactiques sont couramment employés pour déclencher la FML mais l'ensemencement direct du vin se traduit souvent par une forte mortalité des cellules. Il a cependant été montré qu'un prétraitement des cellules d'O. oeni à $42^{\circ} \mathrm{C}$ entraîne une augmentation de leur survie dans le vin, de leur capacité à réaliser la FML et s'accompagne de la synthèse de protéines de stress (Hsp) [10].

Chez les bactéries lactiques, l'étude de la réponse au stress est assez récente et l'espèce la mieux caractérisée est Lactococcus lactis. Parmi les Hsp repérées chez L. lactis sur gels bidimensionnels, certaines ont été identifiées par immunodétection comme étant les homologues de DnaK, DnaJ, GroEL, GrpE [1], Lon et la protéase ATP-dépendante ClpB d'Escherichia coli [3]. D'autres Hsp ont été caractérisées au niveau moléculaire. Elles appartiennent aux différents groupes de protéines de stress :

- DnaK [7] et GroEL [15] sont des protéines chaperonnes qui participent au repliement dans une conformation correcte des protéines en cours de synthèse et sont capables de rompre des agrégats protéiques provoqués par un stress ;

- DnaJ [26], GroES [15] et GrpE [7] sont des Hsp assistant les protéines chaperonnes ;

- HflB [18] est une protéase membranaire ATP-dépendante homologue de FtsH dont le rôle chez $E$. coli est de dégrader le facteur $\sigma^{32}$.

Des Hsp de faible poids moléculaire (ou smHsp, de masse allant de 14 à $30 \mathrm{kDa}$ ) ont été repérées chez L. lactis, mais aucune n'a encore été caractérisée.

\section{LES smHsp CHEZ LES BACTÉRIES LACTIQUES}

Récemment, quatre smHsp ont été étudiées chez des bactéries lactiques. Une smHsp de $16 \mathrm{kDa}$ a été purifiée et entièrement séquencée chez Streptococcus thermophilus [8]. D'autres smHsp ont fait l'objet d'études génétiques. Chez Lacto- 
bacillus delbrueckii, le gène d'une smHsp a été partiellement séquencé (EMBL, numéro d'accession P94867). Enfin, deux smHsp sont bien caractérisées au niveau moléculaire : une smHsp de $17 \mathrm{kDa}$ chez Lactobacillus helveticus [25] et Lo18, une smHsp de $18 \mathrm{kDa}$ chez $O$. oeni [14].

Les smHsp sont les protéines de stress les moins conservées dans leur séquence en acides aminés. Cela explique les pourcentages d'identités assez faibles au sein des bactéries lactiques (inférieurs à $38 \%$ ) par rapport aux Hsp de plus haut poids moléculaire qui sont souvent conservées pour plus de $50 \%$ de leur séquence. Les smHsp des bactéries lactiques présentent cependant un motif consensus dans leur région C-terminale (figure 1) et appartiennent à une même famille apparentée aux $\alpha$-cristallines des Eucaryotes. Les $\alpha$ cristallines sont des protéines de stress capables de former in vivo des complexes multimériques allant de 200 à $800 \mathrm{kDa}$. Elles possèdent une activité de chaperonne in vitro comparable aux Hsp de plus haut poids moléculaire [13] : elles limitent l'agrégation des protéines en conditions dénaturantes et favorisent leur renaturation en conditions normales de manière ATP-indépendante. La région C-terminale bien conservée parmi ces protéines semble être impliquée dans cette activité de chaperonne [24] qui est localisée au centre des complexes multimériques [4].

Au sein des procaryotes, il a été montré que chez Mycobacterium tuberculosis, une smHsp de $16 \mathrm{kDa}$ homologue des $\alpha$-cris- tallines est capable de former des complexes multimériques et possède une activité de chaperonne in vitro. La surexpression de cette smHsp limite l'autolyse des cellules lors de la phase stationnaire tardive [27]. Cette smHsp est aussi un des facteurs antigéniques majeurs de $M$. tuberculosis [5].

La localisation cellulaire de Lo18 a été examinée chez $O$. oeni. Elle est détectée à la fois dans la fraction cytoplasmique et la fraction membranaire. Lol8 est reliée de manière périphérique à la membrane plasmique par des interactions protéineprotéine faibles [14]. La détection d'une smHsp à la fois dans le cytoplasme et la membrane plasmique a déjà été rapportée pour la protéine SP21 chez Stigmatella aurantiaca [17].

La membrane plasmique étant la première cible lors d'un stress thermique, acide ou éthanolique, il est possible que la smHsp Lo18 soit impliquée dans la réponse permettant le maintien de l'intégrité de la membrane d' $O$. oeni en participant par son activité de chaperonne à l'adaptation de la structure membranaire aux nouvelles conditions environnementales.

Les facteurs d'induction de ces smHsp sont multiples. Chez O. oeni, Lol8 est fortement surexprimée lors d'un choc thermique à $42{ }^{\circ} \mathrm{C}$ (figure 2). Lors d'un passage rapide de 30 à 37 ou à $42{ }^{\circ} \mathrm{C}$, la protéine est immunodétectée au bout de 5 minutes et pendant au moins 3 heures [14]. Un choc acide (de pH 5,3 à 3 ) ou
O oeni

I delbru

S thermo
RSERRYGNYSRSYYVPGVDENKISAKYENGILNITLPR-SDESQTHHIEIQ
RQERSEGSVSRSESLPNVDKKGISAKLDGGVLTVTLPKVAPEENADTITIE RSERSLTSVQRQYLLENVKEDEIKASYD-GVLKVTIPKDSNKEIKKSISIE RSER

Figure 1. Alignement des séquences C-terminales des smHsp des bactéries lactiques Oenococcus oeni $(O$. oeni), Lactobacillus delbrueckii ( $L$. delbru) et Streptococcus thermophilus (S. thermo). * Acides aminés identiques. La séquence consensus caractéristique des $\alpha$-cristallines est encadrée.

Figure 1. Alignement of $\mathrm{C}$-terminal sequences from lactic acid bacteria Oenococcus oeni (O. oeni), Lactobacillus delbrueckii ( $L$. delbru) and Streptococcus thermophilus ( $S$. thermo). * Identical amino-acids. The $\alpha$-crystallin characteristic consensus sequence is boxed. 


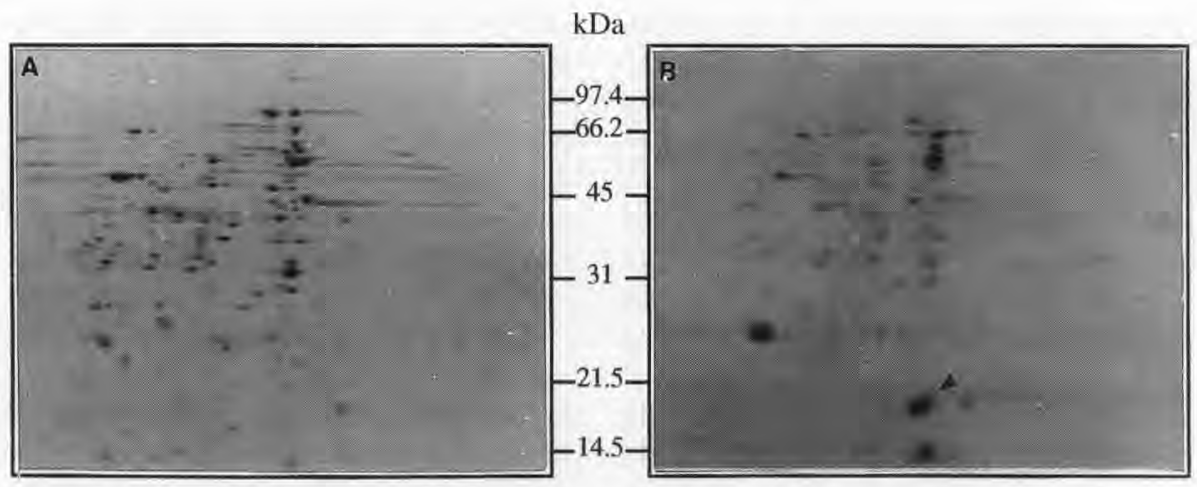

Figure 2. Électrophorèse bidimensionnelle des protéines de Oenococcus oeni marquées à la methionine $\left.{ }^{35} \mathrm{~S}\right]$. A. Conditions normales de croissance. B. Après un choc thermique (1 heure à $\left.42{ }^{\circ} \mathrm{C}\right)$.

Figure 2. Bidimensional electrophoresis of $\left[{ }^{35} \mathrm{~S}\right]$ methionine labelled proteins from Oenococcus oeni. A. Normal growth conditions. B. After a heat shock (1 hour at $\left.42^{\circ} \mathrm{C}\right)$.

éthanolique (éthanol à $12 \% \mathrm{vol} / \mathrm{vol}$ ) ainsi que le passage en phase stationnaire de croissance sont aussi des phénomènes induisant la synthèse de Lo18 [11]. Cette smHsp est donc une composante importante de la réponse globale au stress de $O$. oeni. Chez $S$, thermophilus, la smHsp de $16 \mathrm{kDa}$ est induite par un choc acide. Sa surexpression lors du passage en phase stationnaire de croissance semble être due à l'acidité et non à la carence nutritionnelle du milieu [8]. Chez L, helveticus, la smHsp de $17 \mathrm{kDa}$ est induite lors d'un choc thermique [25].

\section{RÉGULATION DE \\ L'EXPRESSION DES smHsp DES BACTÉRIES LACTIQUES}

Les gènes de smHsp clonés chez $O$. oeni et $L$. helveticus (respectivement $h s p 18$ et $h s p 17$ ) ont été séquencés et leurs régions promotrices déterminées $[14,25]$. Les cadres de lecture de ces gènes sont précédés par des promoteurs caractéristiques de type domestique dits « végétatifs ». Le gène $h s p 17$ de $L$. helveticus possède une séquence inversée-répétée (CIRCE) que l'on retrouve en amont de tous les gènes de protéines de stress connus chez L. lactis (pour revue, [2]). Aucune séquence similaire n'est présente en amont du gène $h s p 18$ chez $O$. oeni.

La comparaison de ces deux régions promotrices identifiées ne permet pas de proposer un mécanisme unique de régulation des gènes de smHsp chez les bactéries lactiques. Les données dont nous disposons pour l'instant sont à comparer aux deux modèles bactériens pour la régulation de l'expression des gènes de stress : E. coli et Bacillus subtilis (pour revue, [21]).

Le mécanisme de régulation établi chez E. coli ne peut pas servir de modèle pour les smHsp des bactéries lactiques. En effet, la réponse au stress $d^{\prime} E$. coli est sous le contrôle des facteurs sigma alternatifs $\sigma^{32}$ et $\sigma^{24}$ qui reconnaissent des promoteurs de type heat shock.

Chez B. subtilis, il existe trois classes de régulation des gènes de stress.

La classe I regroupe les opérons dnaK et $g r o E$ qui possèdent un promoteur végétatif et une séquence CIRCE stabilisée par le répresseur HrcA (figure $3 A$ ). L'inactivation du répresseur lors d'un stress déstabiliserait la séquence inversée-répétée et 
permettrait la transcription des gènes (figure $3 B$ ). Ce modèle pourrait être retenu pour expliquer la régulation du gène $h s p 17$ de $L$. helveticus comme celle des gènes de stress connus chez $L$. lactis.

La régulation des gènes de classe II (une quarantaine de gènes de stress) ne peut pas s'appliquer aux gènes de smHsp des bactéries lactiques puisqu'elle fait intervenir un facteur sigma alternatif, $\sigma^{\mathrm{B}}$ qui permet à l'ARN-polymérase de se fixer sur des promoteurs particuliers.

La classe III comprend les gènes des protéases ATP-dépendantes lon, $\operatorname{clp} C$, $c l p P$ et $f t s H$. Ces gènes possèdent un promoteur végétatif sans séquence CIRCE et font intervenir pour leur régulation un ou plusieurs éléments inconnus. Il a été récemment montré que le gène htpG appartient à cette classe et est soumis à une régulation transcriptionnelle négative puisque la délétion d'une partie en amont de son promoteur permet la transcription du gène [20].

L'expression de la smHsp Lol8 chez $O$. oeni apparaît être régulée au niveau transcriptionnel [14]. Le gène $h s p 18$, sous le contrôle d'un promoteur végétatif mais sans séquence CIRCE, semble s'apparenter à cette classe III dont la régulation est encore méconnue. Ce serait aussi le cas des gènes de smHsp d'autres micro-organismes comme Clostridium acetobutylicum [19] ou Streptomyces albus [22].

Toutefois, Lol 8 étant synthétisée en phase stationnaire de croissance, on ne peut exclure qu'un facteur sigma homologue à $\sigma^{s}$ (le facteur sigma alternatif de la

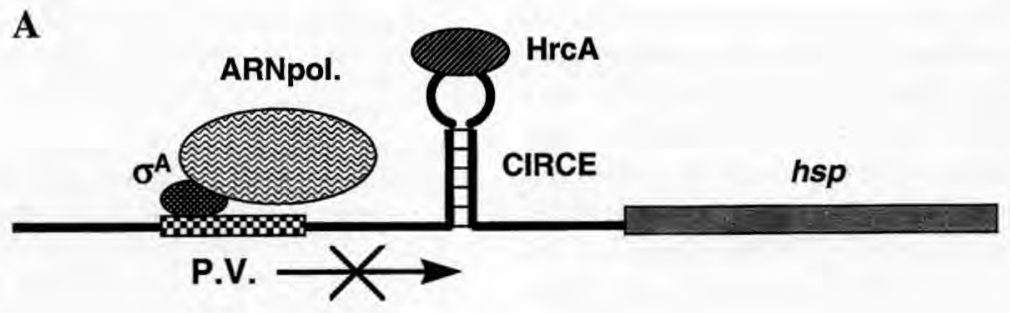

B

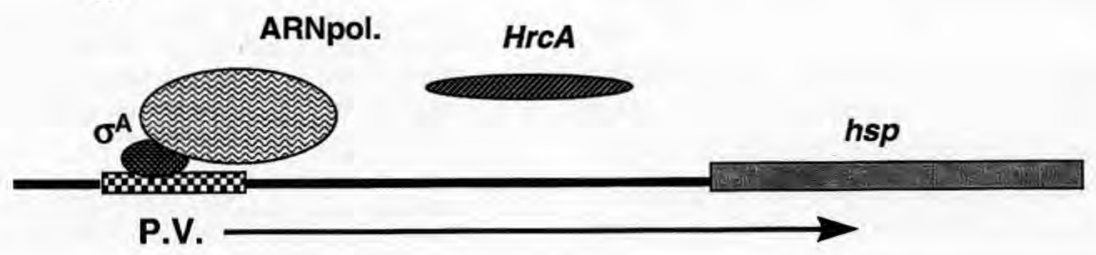

Figure 3. Régulation des gènes de choc thermique de classe I chez Bacillus subtilis. A. Conditions normales de croissance. B. Après choc thermique. $h s p$ : gène de protéine de stress ; P.V.: promoteur végétatif ; $\mathrm{CIRCE}$ : séquence inversée-répétée ; HrcA : régulateur; $\mathrm{HrcA}$ : régulateur dénaturé ; ARN-pol. : ARN-polymérase. La flèche indique l'initiation de la transcription.

Figure 3. Regulation of class I heat-shock genes in Bacillus subtilis. A. Normal growth conditions. B. After thermal shock. $h s p$ : heat-shock gene; P.V.: vegetative promoter; CIRCE: contolling inverted-repeat of chaperone expression; HrcA: regulator; HrcA: denaturated regulator; ARN-pol.: RNA-polymerase. Arrow indicates the trancription inititation. 
phase stationnaire chez E. coli [12]) soit impliqué dans l'expression du gène, même si à l'heure actuelle encore aucun facteur sigma alternatif n'a été mis en évidence chez une bactérie lactique.

Une régulation post-transcriptionnelle pourrait aussi intervenir au niveau de la stabilité du messager, de l'efficacité de sa traduction ou de la stabilité de la protéine. Ainsi, chez $S$. albus, la synthèse de la smHsp Hspl 18 est soumise d'une part à une régulation transcriptionnelle faisant intervenir un régulateur négatif, d'autre part à une thermorégulation post-transcriptionnelle agissant sur l'efficacité de traduction du messager [23].

\section{CONCLUSION}

L'étude de la réponse au stress chez les bactéries lactiques devrait permettre d'accroître la résistance des cellules aux contraintes imposées dans leur environnement en vue d'améliorer la qualité des levains utilisés pour l'ensemencement des milieux extrêmes. Cependant, si les diverses Hsp de haut poids moléculaire sont relativement bien caractérisées, les connaissances acquises sur les smHsp sont encore restreintes. Ce sont les Hsp les moins conservées au niveau de leur séquence en acides aminés. Leurs facteurs d'induction sont multiples et varient selon les microorganismes. Enfin, les mécanismes de régulation de leur expression ainsi que leur rôle dans la réponse au stress constituent de larges champs à explorer.

\section{REMERCIEMENTS}

Ces travaux ont bénéficié du soutien financier du Conseil Régional de Bourgogne et de l'Anvar de Bourgogne.

\section{RÉFÉRENCES}

[1] Auffray Y., Gansel X., Thammavongs B., Boutibonnes $\mathrm{P}$., Heat shock-induced protein synthesis in Lactococcus lactis subsp lactis, Curr. Microbiol. 24 (1992) 281-284.

[2] Boutibonnes P., Les protéines de choc thermique chez Lactococcus lactis : synthèse et régulation ; thermotolérance, Lait 76 (1996) 111-128.

[3] Boutibonnes P., Bisson V., Thammavongs B., Hartke A., Panoff J.M., Benachour A., Auffray $\mathrm{Y}$., Induction of thermotolerance by chemical agents in Lactococcus lactis IL 1403 , Int. J, Food Microbiol. 25 (1995) 83-94.

[4] Boyle D., Gopalakrishnan S., Takemoto L., Localization of the chaperone binding site, Biochem. Biophys. Res. Commun. 192 (1993) 1147-1154.

[5] Chang Z., Primm T.P., Jakana J., Lee I.H, Serysheva I., Chiu W., Gilbert H.F., Quiocho F.A., Mycobacterium tuberculosis 16-kDa antigen (Hsp 16.3) functions as an oligomeric structure in vitro to supress thermal aggregation, J. Biol. Chem. 271 (1996) 7218-7223.

[6] Dicks L.M., Dellaglio F., Collins M.D., Proposal to reclassify Leuconostoc oenos as Oenococcus oeni (corig.) gen. nov., comb. nov., Int. J. Syst. Bacteriol. 45 (1995) 395-397.

[7] Eaton T., Shearman C., Gasson M., Cloning and sequence analysis of the $d n a K$ gene region of Lactococcus lactis subsp. lactis, J. Gen. Microbiol. 139 (1993) 3253-3264.

[8] Gonzalez-Marquez H., Réponse au stress acide chez Streptococcus thermophilus. Purification, identification et caractérisation d'une protéine surexprimée, $\mathrm{Ph}$. D. Thesis, université Henry-Poincaré, Nancy, France, 1997

[9] Gonzalez-Marquez H., Perrin C., Bracquert P., Guimont C., Linden G., A 16 kDa protein family overexpressed by Streptococcus thermophilus PB18 in acid environments, Microbiology 143 (1997) 1587-1594.

[10] Guzzo J., Cavin J.F., Diviès C., Induction of stress proteins in Leuconostoc oenos to perform direct inoculation of wine, Biotechnol. Lett. 16 (1994) 1189-1194.

[11] Guzzo J., Delmas F., Pierre F., Jobin M.P., Samyn B., Van Beeumen J., Cavin J.F., Diviès C., A small heat shock protein from Leuconostoc oenos induced by multiple stresses and during stationary growth phase, Lett. Appl. Microbiol. 24 (1997) 393-396.

[12] Hengge-Aronis R., Survival of hunger and stress: The role of rpoS in early stationary phase gene regulation in Escherichia coli, Cell 72 (1993) 165-168. 
[13] Horwitz $\mathbf{J}_{\text {., }} \boldsymbol{\alpha}$-Crystallin can function as a molecular chaperone, Proc. Natl. Acad. Sci. U.S.A. 89 (1992) 10449-10453.

[14] Jobin M.P., Delmas F., Garmyn D., Diviès C., Guzzo J., Molecular characterization of the gene encoding an 18-kilodalton small heat shock protein associated with the membrane of Leuconostoc oenos, Appl. Environ. Microbiol. 63 (1997) 609-614.

[15] Kim S.G., Batt C.A., Cloning and sequencing of the Lactococcus lactis subsp lactis GroESL operon, Gene 127 (1993) 121-126.

[16] Kunkee R.E., Some roles of malic acid in the malolactic fermentation in wine making, FEMS Microbiol. Rev. 88 (1991) 55-72.

[17] Lünsdorf H., Schairer H.U., Heidelbach M., Localization of the stress protein SP21 in indole-induced spores, fruiting bodies, and heat-shocked cells of Stigmatella aurantiaca, J. Bacteriol. 177 (1995) 7092-7099.

[18] Nilsson D., Lauridsen A.A., Tomoyasu T., Ogura T., A Lactococcus lactis gene encodes a membrane protein with putative ATP-ase activity that is homologous to the essential Escherichia coli ftsH gene product, Microbiology 140 (1994) 2601-2610.

[19] Sauer U., Dürre P., Sequence and molecular characterization of a DNA region encoding a small heat shock protein of Clostridium acetobutylicum, J. Bacteriol. 175 (1993) 3394-3400.

[20] Schulz A., Schwab S., Homuth G., Versteeg S., Schumann W., The htpG gene of Bacillus subtilis belongs to class III heat shock genes and is under negative control, J. Bacteriol. 179 (1997) 3103-3109.
[21] Schumann W., Regulation of the heat shock response in Escherichia coli and Bacillus subtilis, J. Biosci. 21 (1996) 133-148.

[22] Servant P., Mazodier P., Characterization of Streptomyces albus 18-kilodalton heat shockresponsive protein, J. Bacteriol. 177 (1995) 2998-3003.

[23] Servant P., Mazodier P., Heat induction of hsp 18 gene expression in Streptomyces albus G: transcriptional and posttranscriptional regulation, J. Bacteriol. 178 (1996) 7031-7036.

[24] Takemoto L., Emmons T., Horwitz J., The $\mathrm{C}$-terminal region of $\alpha$-crystallin: involvement in protection against heat-induced denaturation, Biochem. J. 294 (1993) 435-438.

[25] Timpone D.E., Fenster K.M., Wei L., Broadbent J.R., Steele J.L., A small heat shock protein from Lactobacillus helveticus, In: G. Venema, J.H.J. Huis in't Veld, J. Hugenholtz (Eds), Abstracts of the Fifth symposium on lactic acid bacteria: genetics, metabolism and applications, Veldhoven, The Netherlands, 1996.

[26] Van Asseldonk A., Simmons A., Visser H., de Vos W.M., Simmons G., Cloning, nucleotide sequence and regulatory analysis of the Lactococcus lactis dnaJ gene, Gene Dev. 1 (1993) 525-531

[27] Yuan Y., Crane D.D., Barry C.E. III, Stationary phase-associated protein expression in Mycobacterium tuberculosis: function of the mycobacterial $\alpha$-crystallin homolog, J. Bacteriol. 178 (1996) 4484-4492. 